\title{
Fabrication and Characterization of Nylon 6/Cellulose Nanofibrils Melt-spun Nanocomposite Filaments
}

Rui Zhu ${ }^{\text {a }}$, Vikram Yadama ${ }^{\text {b }}$, Hang Liu ${ }^{\text {c }}$, Richard J.T. Lin ${ }^{\text {d }}$, David P. Harper ${ }^{\text {e }}$

\begin{abstract}
Nylon 6/cellulose nanofibrils (CNFs) melt-spun nanocomposite filaments were melt spun using a capillary rheometer to explore their capacity as textile materials with potentially improved fabric comfort. The effects of CNF loading level $(0-10 \mathrm{wt} \%)$ on the morphological structures, mechanical and physical properties of the nanocomposite filaments were evaluated. The nanocomposite filaments have much rougher surfaces and non-uniform diameters compared to neat Nylon 6 filaments. Nanoindentation test on the cross-section of the filaments revealed that there was no significant agglomeration of CNFs. As the CNF loading level was increased, the complex viscosity and storage modulus of the nanocomposite filaments were increased, whereas thermal stability was retained. Tenacity and initial modulus of the nanocomposite filaments were improved due to excellent mechanical properties of CNFs. The incorporation of CNFs also increased the
\end{abstract}


hydrophilicity of the nanocomposite filaments, which could have positive implications for textiles as it could potentially improve the perceived comfort.

\section{Keywords:}

A. Cellulose; A. Nanocomposites; B. Surface properties; E. Melt-spinning.

\section{Introduction}

Nanocomposite defines a two-phase material where one of the phases (nanofillers) has at least one dimension in nanometer range (1-100 nm) [1]. Incorporating nanofillers in polymer matrices significantly improve the material properties of virgin polymer. However, most of the nanofillers, such as carbon nanotubes, are expensive to produce, difficult to recycle and not biodegradable. Recently, the use of nanocelluloses as reinforcements in polymers has drawn growing research attention. Compared to other nanofillers, using nanocelluloses as reinforcement offers distinct environmental advantages and biological compatibility [2]. Between the two families of nanocelluloses (cellulose nanofibrils (CNFs) and cellulose nanocrystals (CNCs)), CNFs can be more easily produced with high yield, which makes them a viable replacement to CNCs in reinforcing polymer matrix $[3,4]$. CNFs have diameter in the range of 10 to $100 \mathrm{~nm}$ and length on the order of microns. CNFs are generally produced by disintegration of wood pulp through an intensive mechanical process, such as refining and homogenizing, microfluidization, grinding, cryocrushing and high intensity ultrasonication [5]. CNFs have outstanding mechanical properties due to their crystalline structure. The modulus of elasticity (MOE) along the chain axis of a perfect crystal of native cellulose was estimated between 130-250 GPa [6]. CNFs also have other 
advantages such as high biocompatibility and reactivity [7].

Nylon 6 or polyamide 6 , is a preferred engineering thermoplastic with high melting temperature $\left(\sim 220^{\circ} \mathrm{C}\right)$, good dimensional stability and excellent chemical resistance. It has been used in various industrial applications such as packaging, automotive, and textiles. Recently, cellulosic fillers have been used to reinforce Nylon 6. However, discouraging results were obtained due to the severe thermal degradation of cellulosic fillers at temperature above $200{ }^{\circ} \mathrm{C}$. Compared to other cellulosic fillers, CNFs are more thermally stable because they are purified from lignin, hemicellulose and other thermally labile constituents [8]. Since both Nylon 6 and CNFs are polar materials, it is also believed that they will be compatible with each other leading to a good interaction between them.

Melt spinning is one of the most economical and convenient techniques for manufacturing continuous man-made fibers in large quantities in textile industry [9]. Different techniques have been continuously developed to improve the processability and properties of the spun fibers [10]. Conventional fillers such as glass fiber are not suitable to be used as reinforcing fillers for melt-spun fibers due to the small hole diameter of the spinneret. Incorporating nanofillers such as CNFs into polymer matrix is an alternative approach for reinforcing spun fibers. Since incorporation of nano-fillers comes at a cost, there has to be a compelling reason for it. For instance, Nylon yarn is widely used for protective clothing in the textile industry; however, a common complaint with Nylon is that it is not comfortable to wear. Fabric comfort is often associated with their hydrophilic properties, where yarns that tend to be hydrophilic provide a better feel and are more 
breathable [11]. Since CNFs are hydrophilic in nature, it is hypothesized that addition of CNFs would improve the hydrophilic properties of the Nylon yarn. As per our knowledge, very limited work has been done to incorporate CNFs in Nylon 6 matrix to fabricate nanocomposite filaments to impart desirable attributes that would improve fabric performance.

The primary objective of this study is to develop a processing scheme to fabricate Nylon 6/CNFs melt-spun nanocomposite filaments with good dispersion of CNFs, and to evaluate the performance of the nanocomposite filaments for their suitability as textile yarn. Scanning electron microscopy and nanoindentation were used to understand the surface morphology and dispersion of CNFs in Nylon 6 matrix. The effects of CNF loading levels on the rheological, thermal, and mechanical properties were explored. Hydrophilic behavior and moisture absorption of the nanocomposite filaments were also evaluated.

\section{Materials and Methods}

\subsection{Materials}

Nylon 6 (Zytel 7335F NC010) was purchased from DuPont Company (Wilmington, DE) $\left(\right.$ density $=1130 \mathrm{~kg} \mathrm{~m}^{-3}$; melting temperature $\left.=221^{\circ} \mathrm{C}\right)$. Spray-dried CNFs in powder form was purchased from Process Development Center (PDC) at the University of Maine.

\subsection{Fabrication of Nylon 6/CNFs nanocomposites}

The detailed scheme of the fabrication process of Nylon 6/CNFs melt-spun nanocomposite filaments is demonstrated in Figure 1. Nylon 6 pellets were first dried in a convection oven at $80{ }^{\circ} \mathrm{C}$ for $\sim 16 \mathrm{~h}$ to avoid moisture induced degradation reactions. A 
masterbatch containing $10 \mathrm{wt} \% \mathrm{CNF}$ in Nylon 6 was compounded to improve the dispersion of CNFs during subsequent processes. Prior to the extruding process, Nylon 6 pellets and CNF were hand mixed in a plastic bag; pre-weighed CNF was gradually added and mixed during this process. Subsequently, this mixture was compounded in a co-rotating twin-screw extruder (Leistriz ZSE-18HP) to produce the masterbatch blend. The diameter and the length-to-diameter ratio (L/D) of the screw were $18 \mathrm{~mm}$ and 40 , respectively. The temperature profile was set at $200,210,220,230,230,230,225,220^{\circ} \mathrm{C}$ from the feed hopper to the die. The screw speed was maintained at $70 \mathrm{rpm}$ and the mean value of the residence time was $\sim 3$ mins. The extrudate was drawn through a cooling water bath and pelletized using a lab scale granulator. The masterbatch was further diluted with virgin Nylon 6 to obtain different CNF loading levels $(2.5,5,7.5,10 \mathrm{wt} \%)$, as shown in Table 1. Finally, the diluted Nylon 6/CNF formulations were compounded using a micro compounder (Thermo Scientific, HAAKE MiniLab II) at $230{ }^{\circ} \mathrm{C}$ and $25 \mathrm{rpm}$. The extruded pellets were dried in an oven at $80^{\circ} \mathrm{C}$ overnight before melt-spinning process. The Nylon $6 /$ CNFs nanocomposite filaments prepared by melt-spinning, as explained in section 2.3, are denoted as Nylon 6-XCNF, where $\mathrm{X}$ is the CNF loading level in wt\%.

\subsection{The production of Nylon 6/CNFs melt-spun nanocomposite filaments}

The capillary rheometer is very similar to piston machine used in the melt spinning process [12]. Nylon 6/CNFs melt-spun nanocomposite filaments were fabricated using a capillary rheometer (Rheometric Scientific, ACER 2000). The barrel diameter and cylinder length are $20 \mathrm{~cm}$ and $350 \mathrm{~cm}$, respectively. The machine was equipped with a $1 \mathrm{~mm}$ die 
with length of $30 \mathrm{~mm}$ and angle of $90^{\circ}$. The Nylon/CNFs nanocomposite pellets $(5 \mathrm{~g})$ obtained in section 2.2 were fed manually from top of the rheometer into the barrel. The melt spinning was carried out at the barrel temperature of $230{ }^{\circ} \mathrm{C}$. Based on preliminary experiments, constant shear rate $(\gamma)$ of $200 \mathrm{~s}^{-1}$ was chosen to ensure sufficient time to crystallize the melt. The piston rate $(v)$ of $3.75 \mathrm{~mm} / \mathrm{min}$ was calculated using the equation below,

where $\mathrm{r}$ and $\mathrm{R}$ are die radius and barrel radius, respectively. The melt-spun filaments were cooled by air with a fan attached to the bottom of the capillary rheometer and then manually collected.

\subsection{Surface morphology}

A field-emission scanning electron microscopy (SEM) (FEI Quanta 200F) was utilized to examine the surface morphology of the nanocomposite filaments. To obtain the longitudinal images, Nanocomposite filaments were cut into short strips and taped onto carbon conductive tabs. All samples were sputter-coated with a 1-2 nm thick conductive layer of gold prior to analysis. The acceleration voltage was $20 \mathrm{kV}$.

\subsection{The dispersion of CNFs in Nylon 6 matrix}

The dispersion of CNFs in the Nylon 6 matrix was analyzed using transmission electron microscopy (TEM). The Nylon 6/CNFs melt-spun nanocomposite filaments were cryosectioned to produce ultra-thin film specimen of $60-80 \mathrm{~nm}$ thick, at $-130{ }^{\circ} \mathrm{C}$. Uranyl acetate $(1 \%)$ was used as a negative stain for better contrast. The specimens were then mounted on 
carbon-coated C-flat grids and examined with a TEM (FEI T20) at an accelerating voltage of $200 \mathrm{kV}$.

Besides TEM, the nanoindentation technique was also employed to study the dispersion of CNFs in the nylon 6 matrix by comparing the differences in material's hardness and modulus at various locations. Nanoindentation tests were conducted on the cross-section of the filaments using a T1950 Hysitron TriboIndenter nanoindentation system with a threeside pyramid (Berkovich) diamond indenter. The filaments were embedded into epoxy resin in a cone shape mold, and then the sample surfaces were polished using microtome. Nine indentations were performed on a $3 \times 3$ square grid for each filament. The distance between the indentations was $20 \mu \mathrm{m}$ to avoid interaction.

\subsection{Rheological properties}

Rheological properties of the neat Nylon 6 and nanocomposite filaments were measured on a stress-controlled oscillatory rheometer (DHR-2, TA Instruments, USA) at a temperature of $230{ }^{\circ} \mathrm{C}$. The rheometer was equipped with $25 \mathrm{~mm}$ diameter parallel plates. A dynamic strain sweep was performed in advance to determine the linear viscoelastic region of the materials, and consequently a strain of $1 \%$ was selected for the frequency sweep test. Dynamic frequency sweep tests were performed from 0.1 to $100 \mathrm{rad} / \mathrm{s}$. Specimens were placed between the preheated parallel plates $\left(230^{\circ} \mathrm{C}\right)$, and were allowed to equilibrate for 2 min prior to each frequency sweep run. The gap distance between the two parallel plates was $1 \mathrm{~mm}$. 


\subsection{Thermal properties}

Differential scanning calorimetry (DSC) (TA instrument DSC 2920) was carried out to study the effect of increasing CNF content on the melting and crystallization behavior of Nylon 6. Specimen $(10 \mathrm{mg})$ cut from the nanocomposite filaments was heated from 30 to $250{ }^{\circ} \mathrm{C}$ at a heating rate of $10{ }^{\circ} \mathrm{C} / \mathrm{min}$ and held at $250{ }^{\circ} \mathrm{C}$ for $2 \mathrm{~min}$. The specimen was then cooled to $30^{\circ} \mathrm{C}$ at a cooling rate of $10{ }^{\circ} \mathrm{C} / \mathrm{min}$ and reheated to $250{ }^{\circ} \mathrm{C}$ at a heating rate of 10 ${ }^{\circ} \mathrm{C} /$ min. This cooling and heating procedure was adopted to ensure complete melting of the crystalline fraction of Nylon 6 and remove any thermal history. A nitrogen flux $(80 \mathrm{ml} / \mathrm{min})$ was used as a purge gas for the furnace. Cold crystallization temperature $\left(T_{\mathrm{cc}}\right)$ was determined from the first cooling scan. Glass transition temperature $\left(T_{\mathrm{g}}\right)$, melt temperature $\left(T_{\mathrm{m}}\right)$, and enthalpy of melting $\left(\Delta H_{\mathrm{m}}\right)$ were determined from the second heating scan. The degree of crystallinity $\left(X_{\mathrm{c}}\right)$ was calculated according to the following equation.

where is the heat of fusion of the nanocomposite filament, is the heat of fusion for Nylon 6 of theoretically $100 \%$ crystallinity $(190 \mathrm{~J} / \mathrm{g})$ [13], and is the mass fraction of Nylon 6 in the nanocomposite filament. The $T_{\mathrm{g}}$ of the nylon $6 / \mathrm{CNF}$ nanocomposite filaments were also calculated by means of the Fox equation [14] to evaluate the specific interactions and miscibility of binary components in the nanocomposites:

where and are the mass percentages of Nylon 6 and CNFs, and are the glass transition temperatures for Nylon 6 and CNFs, respectively. 


\subsection{Tensile properties}

The mechanical properties of the nanocomposite filaments were evaluated using tensile test on a screw-driven universal testing machine (Instron, 5565A) equipped with a $5 \mathrm{kN}$ electronic load cell and mechanical grips. The tensile test was conducted at a crosshead speed of $2 \mathrm{~mm} / \mathrm{min}$ and a gauge length of $20 \mathrm{~mm}$. The linear density (tex) of the filaments was calculated by the following equation:

where $\mathrm{m}$ is weight $(\mathrm{g})$ of the filament randomly selected, and $\mathrm{L}(\mathrm{m})$ is length of the filament. The tex number is commonly used to accurately measure sizes in linear and continuous products such as filaments and fibers. Ten replicates were tested for each formulation to obtain the average values of tenacity (cN/tex), initial modulus (cN/tex) and elongation at break (\%). All specimens were conditioned for 1 week at $23{ }^{\circ} \mathrm{C}$ and $50 \% \mathrm{RH}$ prior to tensile test.

\subsection{Hydrophilicity and moisture absorption}

The hydrophilic behavior of the Nylon 6/CNFs nanocomposites was characterized using a contact angle analyzer (VCA Optima, AST Products, Inc). Nanocomposite pellets were hot-pressed into a film at $230{ }^{\circ} \mathrm{C}$ and dried overnight in a sealed desiccator. Deionized water was automatically dropped onto the surface of the film. Sixty seconds after the placement of water droplets on the specimen surface, images of the water droplets were captured using a high-resolution video camera. Sessile drop contact angle (static) was measured as the angle between the baseline of a water drop and the tangent at the solid- 
liquid boundary. Reproducibility of the results was assessed by conducting the measurements at three different locations for each film.

The moisture absorption behavior of the nanocomposite filaments was measured in an environmental chamber (Russells G-64). Prior to the absorption test, the nanocomposite filaments were dried to $\sim 0 \%$ moisture content in the convection oven at $105{ }^{\circ} \mathrm{C}$ overnight. $\sim 5 \mathrm{~g}$ of nanocomposite filaments in a metal mesh screen were placed in the environmental chamber with $23{ }^{\circ} \mathrm{C}$ and $90 \% \mathrm{RH}$. The weight of the sample was measured every $30 \mathrm{~min}$ for the first 5 hours. After then, the weight of the sample was measured every 12 hours until it reached a constant value. Three replicates were measured for each sample, and average values were reported.

\section{Results and Discussion}

\subsection{Surface Morphology of nanocomposite filaments}

The surface morphologies in the direction of nanocomposite filament's axes are displayed in Figure 2. The neat Nylon 6 filament has very smooth surface and uniform diameter. In contrast, the nanocomposite filaments generally have uneven surfaces and nonuniform diameters. This phenomenon is even more pronounced in the nanocomposite filaments with higher CNF loading levels. Besides, SEM images were taken and compared at three different locations over the filament length for each formulation, and surface unevenness was observed at different locations along the fiber. Apparent agglomerations were also found on the surface of the nanocomposite filaments, especially with increasing

levels of CNF content. CNFs are highly hydrophilic and tend to agglomerate even without 
compounding with Nylon 6 matrix. These CNF clusters protruded on the surface of the filaments, which lead to changes in surface morphology.

\subsection{Dispersion of CNFs in the Nylon 6 matrix}

Dispersion is the key factor in improving the strength of nanocomposites, as homogeneous separation of nanoscale particles lead to greater interfacial contact between the particles and the matrix [15]. TEM images of the cross-sections of neat Nylon 6 and nanocomposite filaments were analyzed to study the dispersion of CNFs in the Nylon 6 matrix. It is very difficult to track CNFs, even at high CNF loading level (10 wt\%) (Figure 3(b)). Therefore, the dispersion of CNFs in the Nylon 6 matrix was not very apparent from TEM images. Similar result was reported in the polylactic acid (PLA)/cellulose nanowhiskers (CNW) nanocomposite system with $5 \mathrm{wt} \% \mathrm{CNW}$ [1]. This is probably due to the fact that cellulose nanofibrils became less thermally stable during polymer processing at high temperature $\left(>230{ }^{\circ} \mathrm{C}\right)$ compared to other nanofillers, such as nanoclay. The formation of crystallized component in the polymer matrix would also make it difficult to observe the nanofillers. Indeed, the TEM images of nanocomposite filaments show well-defined dark lines (highlighted by red cycles), which could be assigned to the boarders of CNFs.

It is very difficult to quantify the dispersion of CNFs using TEM technique as the nanofillers greatly blurred the crystalline texture, even at considerably high magnifications. Therefore, nanoindentation of the filament cross-sections was conducted to quantify the variations of mechanical properties at different locations. The profiles of load versus displacement curves at different locations for neat Nylon 6 (Figure 3(c)) are identical, 
whereas for Nylon 6/CNF composite filaments there was a large variation in behavior (Figure 3(d)). This phenomenon is expected as the localized mechanical properties are affected by the distribution and/or the agglomeration of CNFs. Larger variations of modulus (Figure 3(e)) in composite filaments implies the inclusion and dispersion of CNFs in the Nylon 6 matrix.

\subsection{Rheological properties of nanocomposite filaments}

An oscillatory melt rheology study was carried out to understand the addition of CNFs on the rheological properties of nanocomposite filaments. The complex viscosity $\left(\left|\eta^{*}\right|\right)$ of neat Nylon 6 and nanocomposite filaments as a function of frequency $(\omega)$ is presented in Figure 4(a). The $|\eta *|$ decreased with the increase of frequency for all formulations, indicating a shear thinning behavior. The $\left|\eta^{*}\right|$ of the nanocomposite filaments also increased with the addition of CNFs in the entire frequency range, because higher degree of Nylon 6CNF interactions due to increasing CNF content required higher shear stress and longer relaxation time for the nanocomposite filaments to flow [16]. Besides, the orientation of the CNF molecular chains disturbed the formation of Nylon 6 chain entanglements. The changes of $\left|\eta^{*}\right|$ are much more significant at low frequency, when the hydrodynamic force is very dominant. It is also well known that storage modulus $\left(\mathrm{G}^{\prime}\right)$ at low frequency range is very sensitive to the structural changes in the polymer nanocomposites [17]. Due to the intrinsic rigidity of CNFs, the $\mathrm{G}^{\prime}$ of the nanocomposite filaments was relatively higher than that of neat Nylon 6 at low frequency range (Figure 4(b)). The $G^{\prime}$ of all the formulations became almost identical at frequencies higher than $10 \mathrm{rad} / \mathrm{s}$ due to the hydrodynamic force. 
The distinct change in viscoelastic behavior due to restricted polymer chain mobility in the nanocomposites has been used to identify the formation of percolated network [18]. As indicated by a sudden increase of $\left|\eta^{*}\right|$ at low frequency, rheological percolated threshold was reached between 5-7.5 wt $\%$ CNFs. There were more filler agglomerations beyond this region, which led to the sudden viscosity increase. Therefore, the optimal loading of CNFs for a uniform dispersion in the Nylon 6 matrix is between 5-7.5 wt\%. The rheological response of the nanocomposite is more like a 'pseudo-solid' than a molten liquid above the percolated threshold [19].

\subsection{Thermal properties of nanocomposite filaments}

DSC analysis was conducted to understand the effect of CNF addition on the melting and crystallization behavior of Nylon 6. Typical DSC thermograms (both first cooling and second heating scan) are presented in Figure 5(a)(b). The glass transition temperature $\left(T_{\mathrm{g}}\right)$, cold crystallization temperature $\left(T_{\mathrm{c}}\right)$, melting temperature $\left(T_{\mathrm{m}}\right)$, enthalpies of fusion $\left(\Delta H_{\mathrm{m}}\right)$ and crystallinity have been determined and summarized in Table 2 . The glass transition of the nanocomposite filaments occurred at a slightly higher temperature than that of neat Nylon 6, which was observed at $50.6{ }^{\circ} \mathrm{C}$. Besides, glass transition temperature of the nanocomposite filaments increased gradually as the CNF loading level increased. The higher $T_{\mathrm{g}}$ of the nanocomposite filaments was mainly due to the strong interactions between polar amide groups of Nylon 6 and hydroxyl groups of CNFs, which reduced the segmental mobility of the nanocomposite structure [20]. The consistent increasing trend of $T_{\mathrm{g}}$ also indicates that $\mathrm{CNF}$ is well-dispersed in the Nylon 6 matrix. The $T_{\mathrm{g}}$ measured by experiment 
was also compared to the $T_{\mathrm{g}}$ predicted by the Fox equation for simple mixing, as shown in Figure 5(c). The Fox equation describes the behavior of $T_{\mathrm{g}}$ of ideal mixtures by assuming that the homogeneous and heterogeneous interactions are equivalent [21]. Therefore, conformational changes at the interface of polymer matrix chains and CNFs are responsible for the deviations from the predicted $T_{\mathrm{g}}[22]$. Overall, the Fox equation predicted the changes of $T_{\mathrm{g}}$, especially at higher loading levels. The measured $T_{\mathrm{g}}$ is slightly higher than predicted $T_{\mathrm{g}}$ at all $\mathrm{CNF}$ loading level, primarily due to decreased conformational freedom of the matrix chains with the addition of CNFs. The result also indicates that the two phases are miscible, as two completely immiscible phases would form a free surface and increase the mobility of the matrix chain, thereby resulting in a lower $T_{\mathrm{g}}$ than predicted [22].

An endothermic melting peak of neat Nylon 6 is observed at $222.9{ }^{\circ} \mathrm{C}$, which is correlated with the $\alpha$-form crystals of Nylon 6 [23]. No essential correlation between the CNF loading levels and changes in melting temperature can be established. A single well defined crystallization exotherm peak of neat Nylon 6 was detected at $\sim 191.4{ }^{\circ} \mathrm{C}$ upon cooling, which can be assigned to $\alpha$-form crystals [24]. The crystallinity of the nanocomposite filaments was decreased due to the addition of CNFs. For instance, the crystallinity of neat Nylon 6 is $32.1 \%$ whereas the crystallinity of Nylon 6-10CNF is 26.9 $\%$. Similar results were found in Nylon 6 nanocomposites containing microcrystalline cellulose (MCC) [16] and Nanoclay [25]. It was well known that the large surface of nanofillers acts as nucleation sites for crystallization [26]. The nucleating effect contributes significantly to the occurrence of transcrystalline layers around the nanofillers, resulting in 
an increase in the degree of crystallinity [27]. However, nanofillers also restrict the mobility of polymer molecular chains and act as barriers affecting crystal growth [28]. In the case of $\mathrm{CNF}$ as nanofillers, the latter became the predominant factor in affecting the crystallization of Nylon 6.

\subsection{Mechanical performance of nanocomposite filaments}

The tensile properties of the melt-spun nanocomposite filaments are shown in Figure 6.

Different letters in the parenthesis mean the values are significantly different from each other. The confidence level was set at $95 \%$. Note that the obtained filaments were meltspun without drawing, therefore, the tenacity and initial modulus reported here are inferior compared to drawn filaments with higher polymer chain orientation and crystallinity. Oneway ANOVA statistical analysis was applied to evaluate the data at the $95 \%$ confidence level. The $p$-value $(0.023)$ of tenacity is less than 0.05 , indicating that the addition of CNFs increased the tenacity of the nanocomposite filaments. The tenacity of Nylon6/CNFs meltspun nanocomposite filaments was in the range of $40.72-50.24 \mathrm{cN} / \mathrm{tex}$. Generally, the tenacity increased as the CNF loading level increased (Figure 6(a)); for instance, the nanocomposite filaments containing $10 \mathrm{wt} \% \mathrm{CNFs}$ showed a tenacity of $50.24 \mathrm{cN} / \mathrm{tex}$, which is $23.4 \%$ increase compared to Neat Nylon 6 with 40.72 cN/tex. Similarly, increasing trend of initial modulus was observed as the CNF loading level increased. Due to good chemical compatibility between Nylon 6 and CNFs, stress transfer is efficient from the matrix to the nanofillers, leading to improved tenacity [29]. The increase of tenacity can also be ascribed to the presence of rigid nanoparticles limiting polymer molecules motion. 
The elongation at break of the nanocomposite filaments was dramatically decreased with the increase of CNF loading level. The filaments were melt-spun without further drawn, therefore considerably higher elongation at break was obtained compared to literature using drawn fibers due to lack of orientation of the molecular chain in the meltspun filament [30]. The elongation at break decreased from $153.21 \%$ to $112.54 \%$ and 69.5 $\%$ with the addition of 5 and $10 \mathrm{wt} \%$ of CNFs, respectively. Similar result using cellulose nanowhiskers as nanofillers in melt-spun polylactic acid fibers has been reported [27]. This could be attributed to limited movement of polymer molecular chains with increasing CNF levels. The agglomerations of CNFs could also lead to poor interaction between Nylon 6 matrix and CNFs, and thus decrease the elongation of nanocomposite filaments.

\subsection{Interaction of nanocomposite filaments to moisture}

Understanding the hydrophilic behavior of the nanocomposite filaments has significant impact on its application as textile materials where comfort is critical [11]. The contact angle is an important parameter to measure the surface hydrophilicity of materials [31]. As shown from Figure 7(a), neat Nylon 6 film exhibited a contact angle of $\sim 72.9^{\circ}$ and was relatively hydrophilic due to the presence of carboxyl groups at the end of its polymer chain. A similar result was reported for water contact angle on Nylon film [32]. The Nylon 6/CNFs nanocomposites had lower contact angle compared to neat Nylon 6 . The contact angle of nanocomposites decreased progressively with respect to the increase in CNF content, e.g, the contact angle of nylon 6-10 CNF was $61.6^{\circ}$, which was much smaller than that of neat nylon 6 statistically. Note that values with different letters mean they were 
significantly different from each other. The decreased contact angle can be attributed to several reasons. Firstly, the contact angle of neat CNFs was reported to be $\sim 41.2^{\circ}[33]$, Therefore, increasing CNF's compositions in the system would decrease the contact angle of the nylon 6/CNF nanocomposites. Secondly, The addition of CNFs also increased the hydrogen bonding sites to react with moisture. Moreover, nanocelluloses have higher polarity and total surface energy than common polymers [34]. Both characteristics make the nanocomposite film more hydrophilic than the neat polymer film [35].

Moisture absorption of the nanocomposite filaments was measured at $23{ }^{\circ} \mathrm{C}$ and $90 \%$ $\mathrm{RH}$ environment. Typical plots of the moisture content of the filaments versus time during moisture absorption test are shown in Figure 7(b). The moisture absorption of the nanocomposites showed an overall increasing trend with the addition of CNFs. The impacts of CNFs on moisture absorption became significant after $5 \mathrm{wt} \%$ CNFs was added into the Nylon 6 matrix. After exposing to the humid conditions for 5 h, Nylon 6-7.5CNF showed the highest moisture content of $4.91 \pm 0.02 \%$, which is $\sim 16 \%$ increase compared to that of neat Nylon $6(4.23 \pm 0.03 \%)$. Overall, the moisture absorption behaviors of nanocomposite filaments agree well with contact angle results. Hydroxyl groups in the cellulose can readily form hydrogen bonds with water and affect the polarity of the main polymer chains, resulting in increased water absorption. The increased hydrophilicity and moisture absorption filaments indicate that the fabrics made from the Nylon 6/CNFs nanocomposite filaments can transport the humidity to the atmosphere faster than neat Nylon 6. Overall, considering various characteristic changes, $7.5 \mathrm{wt} \%$ is the optimal CNF loading level for 
Nylon 6 based polymer matrix. At this loading level, less CNFs agglomeration were formed while considerably high mechanical properties and hydrophilicity were attained.

\section{Conclusions}

Nylon 6/CNFs melt-spun nanocomposite filaments with loading level of CNFs varied in the range of $2.5-10 \mathrm{wt} \%$ were fabricated using a three-step process: masterbatch preparation, melt compounding and melt spinning. The SEM micrographs showed that the addition of CNFs increased the surface roughness of the filaments primarily due to the uneven thermal shrinkage induced by CNFs. The complementary results from TEM images and nanoindentation data could indirectly suggest some degree of CNF dispersion in the nanocomposite filaments. The incorporation of CNFs in Nylon 6 matrix increased complex viscosity and storage modulus, which indicate the formation of percolated CNFs network. The presence of CNFs reduced the segmental mobility of Nylon 6 polymer chain, thereby decreasing the crystallinity of nanocomposite filaments. The moisture absorption of the nanocomposite filaments increased with the addition of CNFs predominantly due to the highly hydrophilic nature of cellulose. In conclusion, by incorporating renewable and lowcost CNFs into Nylon 6 filaments could produce composite filaments with improved multifunctional properties, such as higher tenacity and hydrophilicity, suitable for novel textile applications on consumer and technical end uses.

\section{Acknowledgments}

This work, as part of the Northwest Advanced Renewables Alliance (NARA), was supported by the Agriculture and Food Research Initiative Competitive Grant (number 2011-68005-30416) from the United States Department of Agriculture (USDA) National Institute of Food and Agriculture. 


\section{References}

[1] Oksman K, Mathew AP, Bondeson D, Kvien I. Manufacturing process of cellulose whiskers/polylactic acid nanocomposites. Composites Science and Technology 2006;66:2776-84.

[2] Pei A, Malho J-M, Ruokolainen J, Zhou Q, Berglund LA. Strong Nanocomposite Reinforcement Effects in Polyurethane Elastomer with Low Volume Fraction of Cellulose Nanocrystals. Macromolecules 2011;44:4422-7.

[3] Chaker A, Mutje P, Vilaseca F, Boufi S. Reinforcing potential of nanofibrillated cellulose from nonwoody plants. Polym Compos 2013;34:1999-2007.

[4] Lu J, Askeland P, Drzal LT. Surface modification of microfibrillated cellulose for epoxy composite applications. Polymer 2008;49:1285-96.

[5] Abdul Khalil HPS, Davoudpour Y, Islam MN, Mustapha A, Sudesh K, Dungani R, et al. Production and modification of nanofibrillated cellulose using various mechanical processes: A review. Carbohydrate Polymers 2014;99 IS -:649-65.

[6] Sakurada I, Nukushina Y, Ito T. Experimental determination of the elastic modulus of crystalline regions in oriented polymers. Journal of Polymer Science 1962;57:651-60.

[7] Zimmermann T, Bordeanu N, Strub E. Properties of nanofibrillated cellulose from different raw materials and its reinforcement potential. Carbohydrate Polymers 2010;79:1086-93.

[8] Panaitescu DM, Frone AN, Nicolae C. Micro- and nano-mechanical characterization of polyamide 11 and its composites containing cellulose nanofibers. European Polymer Journal 2013;49:3857-66.

[9] Hooshmand S, Aitomäki Y, Skrifvars M, Mathew AP, Oksman K. All-cellulose nanocomposite fibers produced by melt spinning cellulose acetate butyrate and cellulose nanocrystals. Cellulose 2014;21:2665-78.

[10] O'Brien JP, Aneja AP. Fibres for the next millennium. Review of Progress in Coloration and Related Topics 1999;29:1-7.

[11] Dastjerdi R, Fasahat F. Thermophysiological Comfort by PA6/TiO2 Nanocomposite Yarns, 2013.

[12] Persson M, Lorite GS, Cho S-W, Tuukkanen J, Skrifvars M. Melt Spinning of Poly(lactic acid) and Hydroxyapatite Composite Fibers: Influence of the Filler Content on the Fiber Properties. ACS Appl Mater Interfaces 2013;5:6864-72.

[13] Wu Q, Liu X, Berglund LA. An Unusual Crystallization Behavior in Polyamide 6/ Montmorillonite Nanocomposites. Macromolecular Rapid Communications 2001;22:1438-40.

[14] Fox TG. Influence of diluent and of copolymer composition on the glass temperature of a polymer system. Bull Am Phys Soc 1956;1:123-35.

[15] Mukherjee T, Czaka M, Kao N, Gupta RK, Choi HJ, Bhattacharya S. Dispersion study of nanofibrillated cellulose based poly(butylene adipate-co-terephthalate) 
composites. Carbohydrate Polymers 2014;102 IS -:537-42.

[16] Kiziltas A, Nazari B, Gardner DJ, Bousfield DW. Polyamide 6-Cellulose Composites: Effect of Cellulose Composition on Melt Rheology and Crystallization Behavior. Polym Eng Sci 2014;54:739-46.

[17] Mabrouk AB, Magnin A, Belgacem MN, Boufi S. Melt rheology of nanocomposites based on acrylic copolymer and cellulose whiskers. Composites Science and Technology 2011;71:818-27.

[18] Pegel S, Pötschke P, Petzold G, Alig I, Dudkin SM, Lellinger D. Dispersion, agglomeration, and network formation of multiwalled carbon nanotubes in polycarbonate melts. Polymer 2008;49:974-84.

[19] Mayoral B, Harkin-Jones E, Khanam PN, AlMaadeed MA, Ouederni M, Hamilton AR, et al. Melt processing and characterisation of polyamide 6/graphene nanoplatelet composites. RSC Adv 2015;5:52395-409.

[20] Paci M, Filippi S, Magagnini P. Nanostructure development in nylon 6-Cloisite ${ }^{\circledR}$ $30 \mathrm{~B}$ composites. Effects of the preparation conditions. European Polymer Journal 2010;46:838-53.

[21] Rocco AM, Pereira RP, Felisberti MI. Miscibility, crystallinity and morphological behavior of binary blends of poly(ethylene oxide) and poly(methyl vinyl ethermaleic acid). Polymer 2001;42:5199-205.

[22] Sunday DF, Green DL. Thermal and Rheological Behavior of Polymer Grafted Nanoparticles. Macromolecules 2015;48:8651-9.

[23] Wu TM, Liao CS. Polymorphism in nylon 6/clay nanocomposites. Macromolecular Chemistry and Physics 2000;201:2820-5.

[24] Liu TX, Liu ZH, Ma KX, Shen L, Zeng KY, He CB. Morphology, thermal and mechanical behavior of polyamide 6/layered-silicate nanocomposites. Composites Science and Technology 2003;63:331-7.

[25] Kazemi S, Mojtahedi MRM, Takarada W, Kikutani T. Morphology and crystallization behavior of nylon 6-clay/neat nylon 6 bicomponent nanocomposite fibers. J Appl Polym Sci 2014;131.

[26] Wu D, Wu L, Wu L, Xu B, Zhang Y, Zhang M. Nonisothermal cold crystallization behavior and kinetics of polylactide/clay nanocomposites. Journal of Polymer Science Part B: Polymer Physics 2007;45:1100-13.

[27] John MJ, Anandjiwala R, Oksman K, Mathew AP. Melt-spun polylactic acid fibers: Effect of cellulose nanowhiskers on processing and properties. J Appl Polym Sci 2013;127:274-81.

[28] Xin F, Li L. The role of a silane coupling agent in carbon nanotube/polypropylene composites. Journal of Composite Materials 2012;46:3267-75.

[29] Ozen E, Kiziltas A, Kiziltas EE, Gardner DJ. Natural fiber blend-nylon 6 composites. Polym Compos 2013;34:544-53.

[30] Steinmann W, Walter S, Gries T, Seide G, Roth G. Modification of the mechanical properties of polyamide 6 multifilaments in high-speed melt spinning with nano 
silicates. Textile Research Journal 2012;82:1846-58.

[31] Palacio L, Calvo JI, Prádanos P, Hernández A, Väisänen P, Nyström M. Contact angles and external protein adsorption onto UF membranes. Journal of Membrane Science 1999;152:189-201.

[32] Du J, Luo X, Fu Z, Xu C, Ren X, Gao W, et al. Improving the hydrophobicity of nylon fabric by consecutive treatment with poly(acrylic acid), tetraethylorthosilicate, and octadecylamine. J Appl Polym Sci 2015;132:42456.

[33] Rodionova G, Lenes M, Eriksen Ø, Gregersen Ø. Surface chemical modification of microfibrillated cellulose: improvement of barrier properties for packaging applications. Cellulose 2011;18:127-34.

[34] Khoshkava V, Kamal MR. Effect of Surface Energy on Dispersion and Mechanical Properties of Polymer/Nanocrystalline Cellulose Nanocomposites.

Biomacromolecules 2013;14:3155-63.

[35] Pant HR, Bajgai MP, Nam KT, Seo YA, Pandeya DR, Hong ST, et al. Electrospun nylon-6 spider-net like nanofiber mat containing TiO2 nanoparticles: A multifunctional nanocomposite textile material. Journal of Hazardous Materials 2011;185:124-30.

Figure captions

Figure 1. Scheme of the fabrication process of the Nylon 6/CNFs melt-spun nanocomposite filaments.

Figure 2. SEM images in the longitudinal direction of melt-spun nanocomposite filaments (300 x): (a) neat Nylon 6, (b) Nylon 6-2.5CNF, (c) Nylon 6-5CNF, (d) Nylon 6-7.5CNF, and (e) Nylon 6-10CNF.

Figure 3. TEM images and nanoindentation results: Cross-sectional TEM images of (a) Neat Nylon 6 and (b) Nylon 6-10CNF; Load versus displacement curves of neat 
Nylon 6 (c) and Nylon 6-10CNF (d); Comparison of modulus of neat Nylon 6 and Nylon 6-10CNF at different locations (e).

Figure 4. Complex viscosity (a) and storage modulus (b) as a function of dynamic angular frequency $(\omega)$ of Nylon 6/CNFs melt-spun nanocomposite filaments with various $\mathrm{CNF}$ loading levels at $230{ }^{\circ} \mathrm{C}$.

Figure 5. DSC thermograms of Nylon 6/CNFs melt-spun nanocomposite filaments: (a) second heating scan, (b) first cooling scan. (c) Dependence of $T_{\mathrm{g}}$ on the CNF loading level.

Figure 6. Tensile properties of Nylon 6/CNFs melt-spun nanocomposite filaments: (a) Tenacity and Initial modulus (b) elongation at break.

Figure 7. Water contact angles (a) and moisture absorption behavior (b) of Nylon 6/ CNFs nanocomposite films with respect to the CNF loading levels.

Table 1. Formulations of the Nylon 6/CNFs nanocomposite filaments.

\begin{tabular}{lll} 
& \multicolumn{2}{c}{ Composition (wt\%) } \\
\cline { 2 - 3 } Sample Code & Nylon6 & CNF \\
\hline Neat Nylon 6 & 100 & 0
\end{tabular}




\begin{tabular}{lll} 
Nylon 6-2.5CNF & 97.5 & 2.5 \\
Nylon 6-5CNF & 95 & 5 \\
Nylon 6-7.5CNF & 92.5 & 7.5 \\
Nylon 6-10CNF & 90 & 10 \\
\hline
\end{tabular}

Table 2. Thermal properties of the melt-spun Nylon 6/CNF nanocomposite filaments obtained from DSC thermograms $s^{\mathrm{a}}$.

\begin{tabular}{lccccc}
\hline Sample Code & $T_{\mathrm{g}}\left({ }^{\circ} \mathrm{C}\right)$ & $T_{\mathrm{c}}\left({ }^{\circ} \mathrm{C}\right)$ & $T_{\mathrm{m}}\left({ }^{\circ} \mathrm{C}\right)$ & $\Delta H_{\mathrm{m}}(\mathrm{J} / \mathrm{g})$ & Crystallinity $(\%)$ \\
\hline Neat Nylon 6 & 50.6 & 191.4 & 222.9 & 61.03 & 32.1 \\
Nylon 6-2.5CNF & 52.1 & 191.6 & 223.3 & 56.28 & 30.4 \\
Nylon 6-5CNF & 52.6 & 190.4 & 222.9 & 50.61 & 28.0 \\
Nylon 6-7.5CNF & 52.9 & 190.6 & 224.3 & 48.42 & 27.6 \\
Nylon 6-10CNF & 53.6 & 190.1 & 223.4 & 46.03 & 26.9 \\
${ }^{\mathrm{a}} T_{\mathrm{g}}=$ glass transition temperature, $T_{\mathrm{c}}=$ cold crystallization temperature, and $T_{\mathrm{m}}=$ melting \\
temperature.
\end{tabular}




\section{Figures}

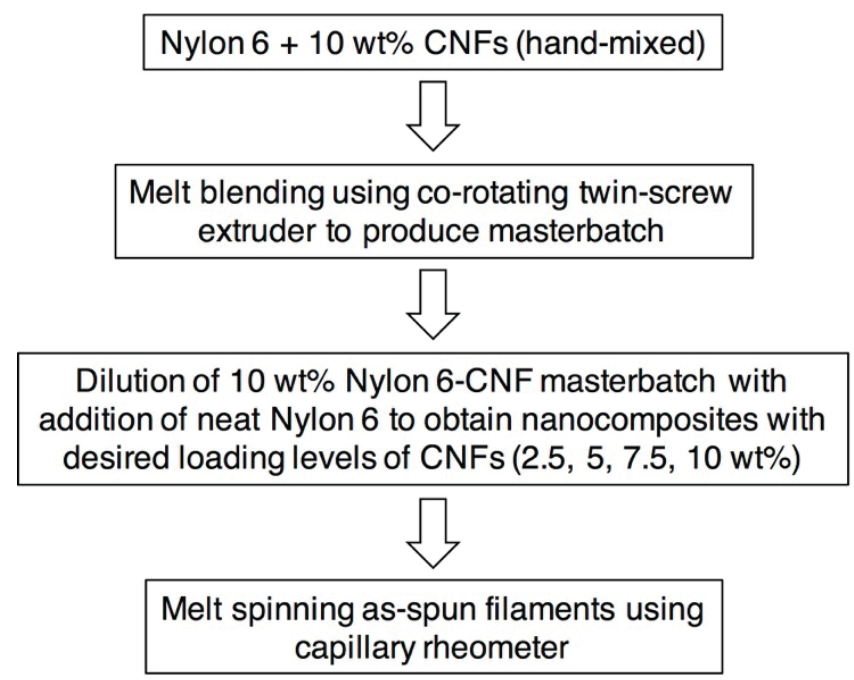

Figure 1. Scheme of the fabrication process of Nylon 6/CNFs melt-spun nanocomposite filaments.
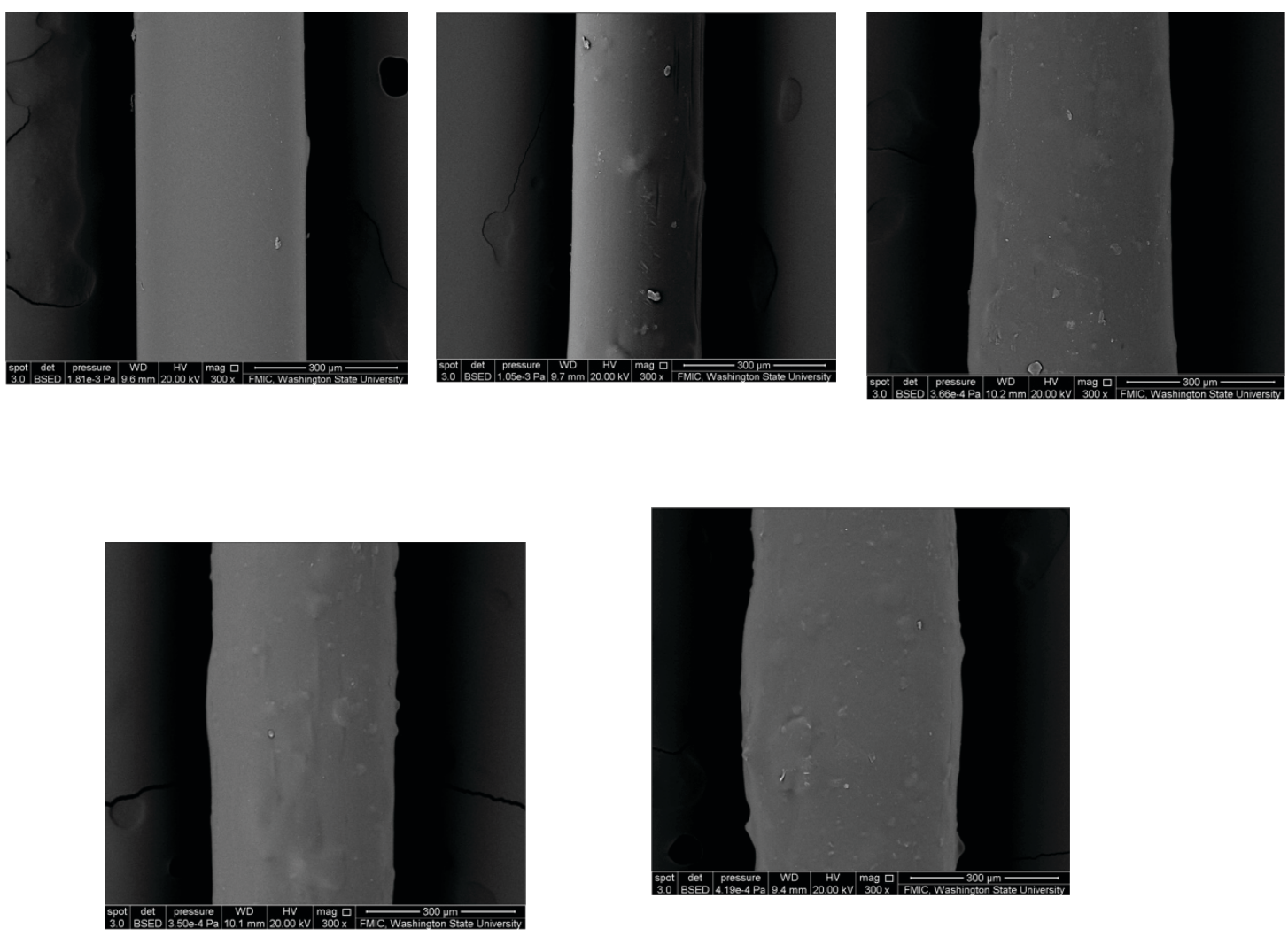
Figure 2. SEM images in the longitudinal direction of melt-spun nanocomposite filaments (300 x): (a) neat Nylon 6, (b) Nylon 6-2.5CNF, (c) Nylon 6-5CNF, (d) Nylon 6-7.5CNF, and (e) Nylon 6-10CNF.

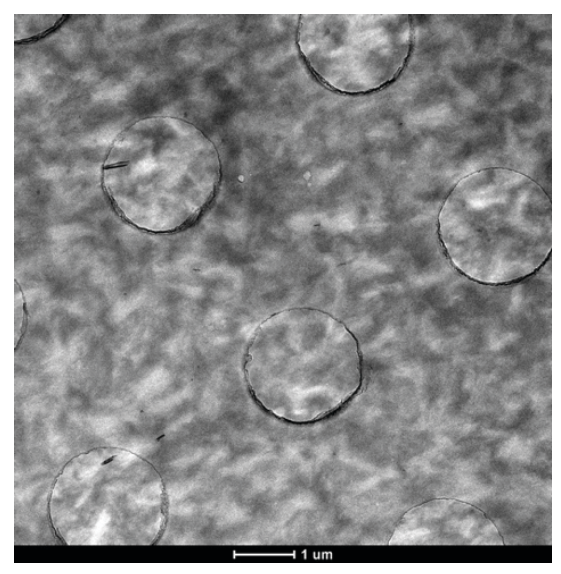

(a)

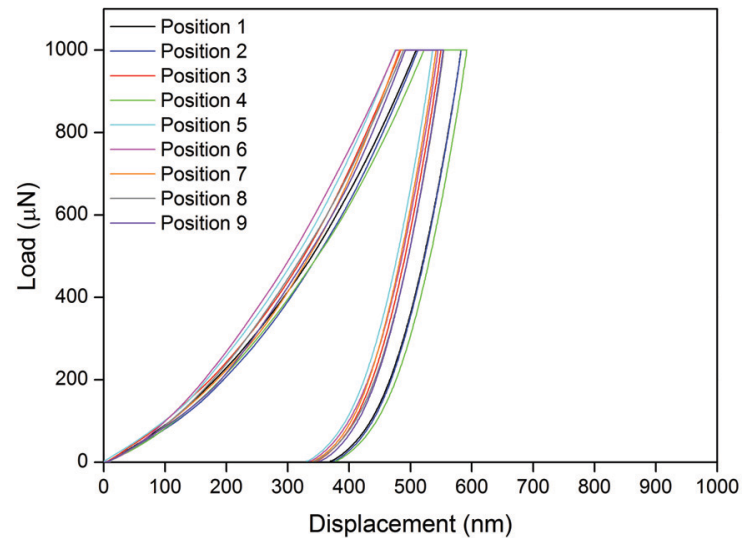

(c)

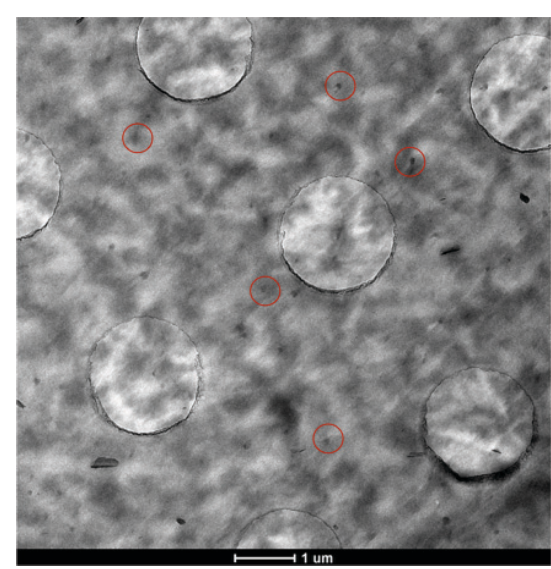

(b)

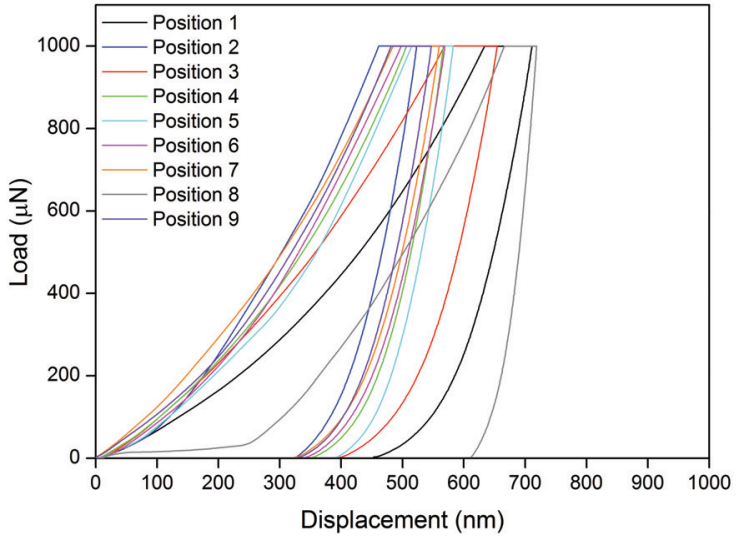

(d)

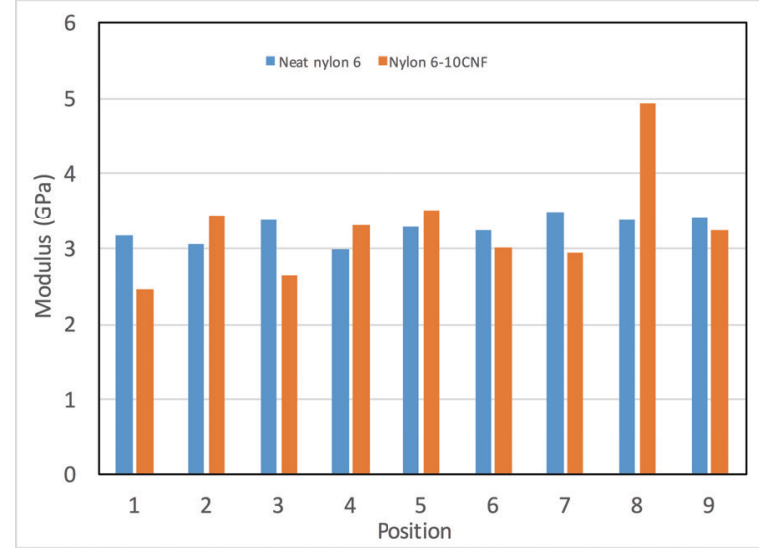


(e)

Figure 3. TEM images and nanoindentation results: Cross-sectional TEM images of (a) Neat Nylon 6 and (b) Nylon 6-10CNF; Load versus displacement curves of neat Nylon 6 (c) and Nylon 6-10CNF (d); Comparison of modulus of neat Nylon 6 and Nylon 6-10CNF at different locations (e).

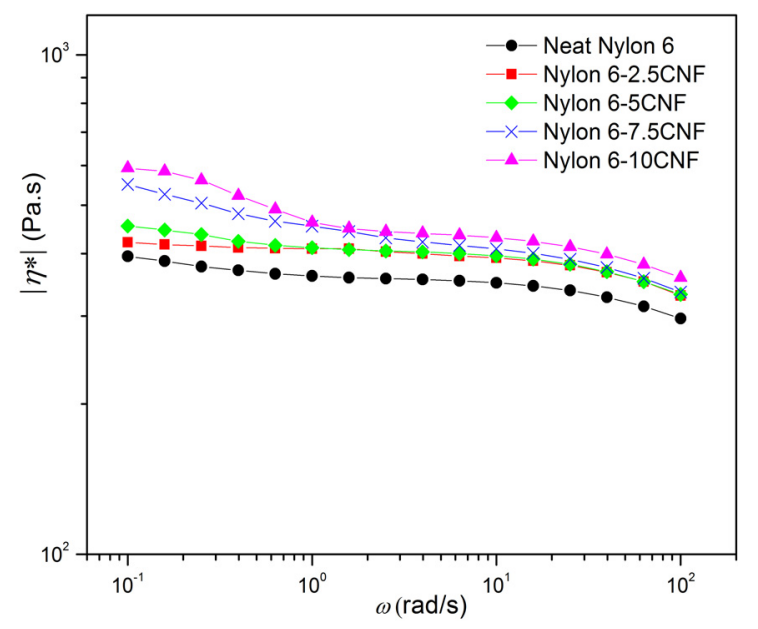

(a)

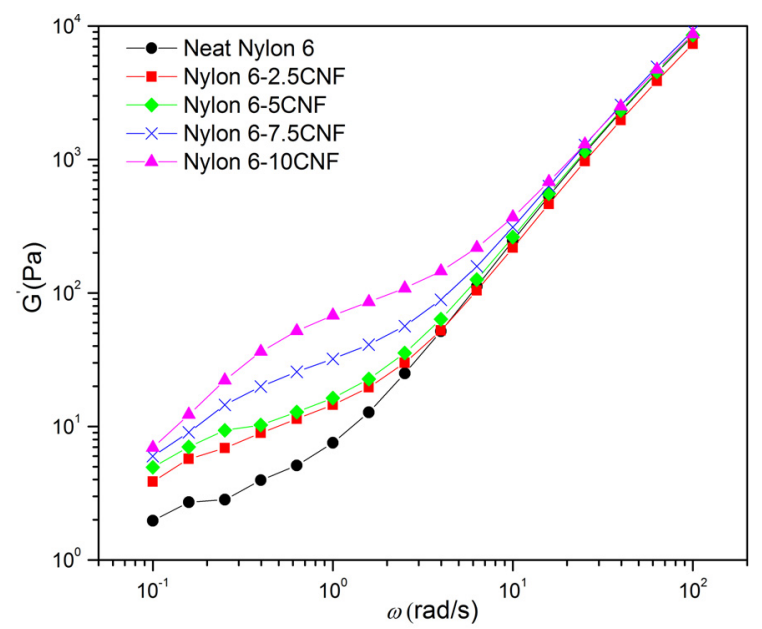

(b)

Figure 4. Complex viscosity (a) and storage modulus (b) as a function of dynamic angular frequency $(\omega)$ of Nylon 6/CNFs melt-spun nanocomposite filaments with various $\mathrm{CNF}$ loading levels at $230{ }^{\circ} \mathrm{C}$. 


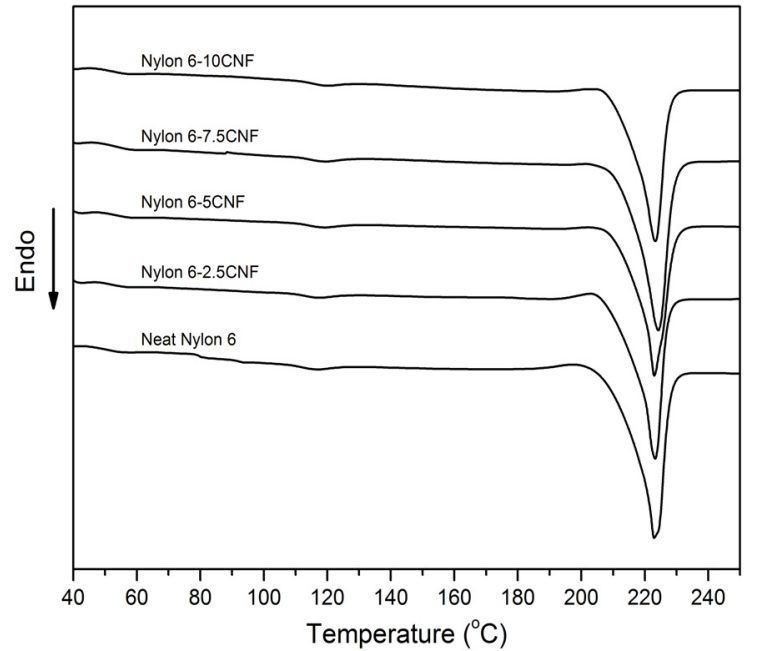

(a)

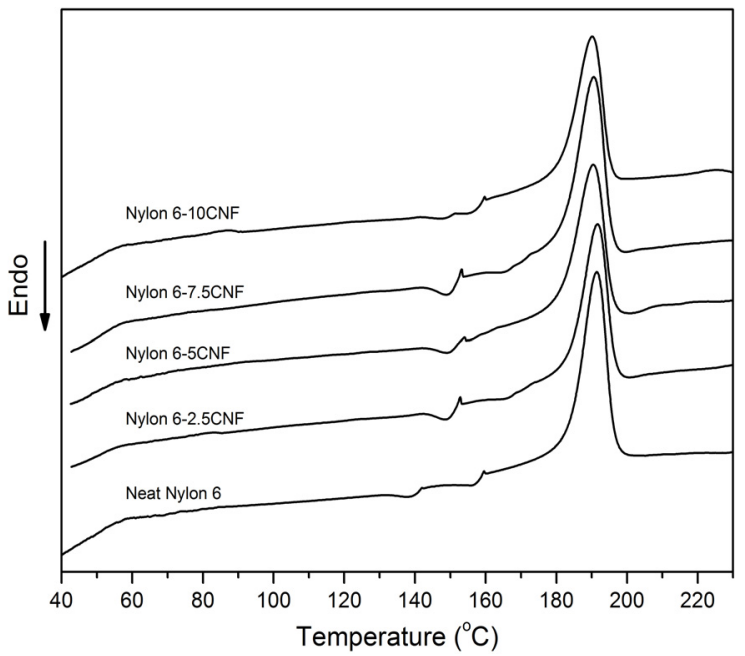

(b) 


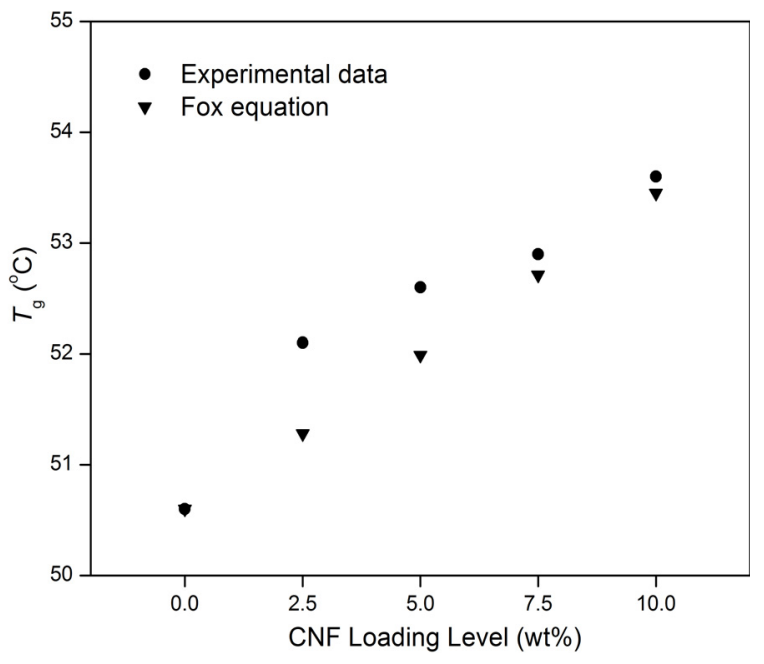

(c)

Figure 5. DSC thermograms of Nylon 6/CNFs melt-spun nanocomposite filaments: (a) second heating scan, (b) first cooling scan. (c) Dependence of $T_{g}$ on the CNF loading level.

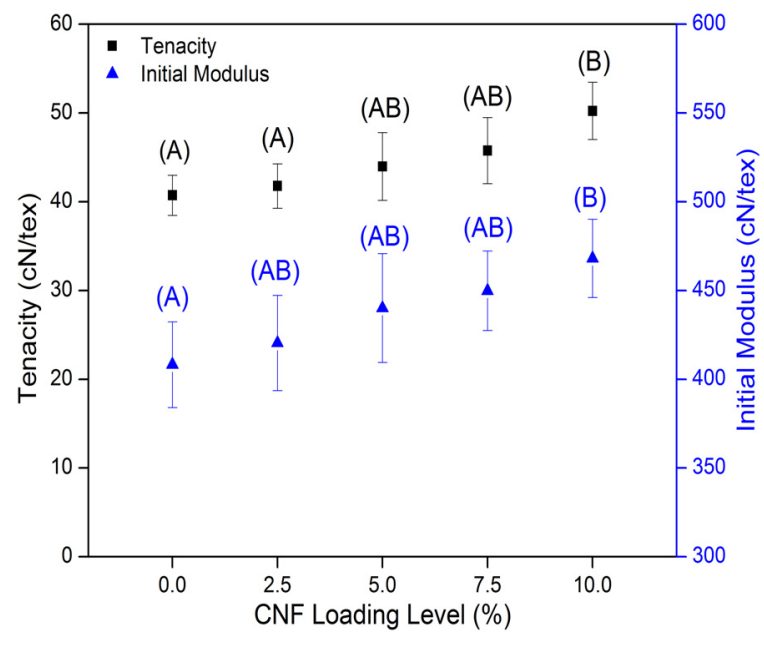

(a)

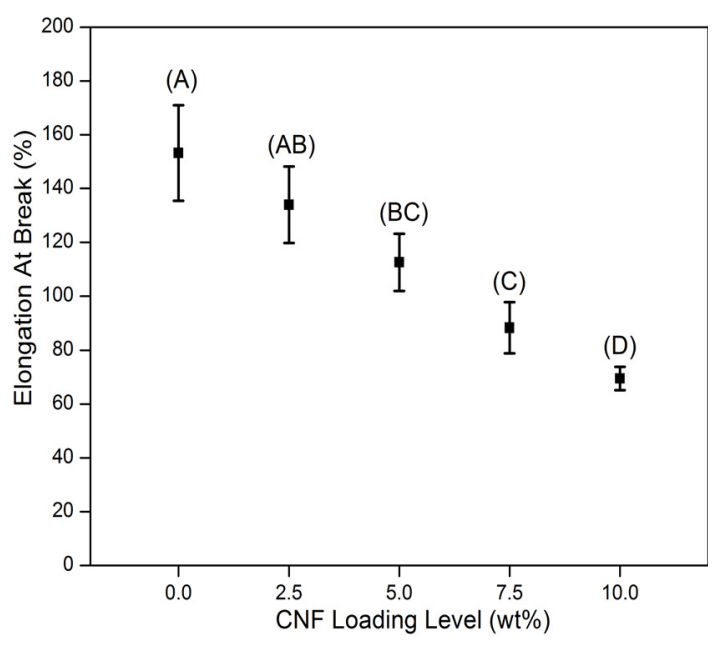

(b)

Figure 6. Tensile properties of Nylon 6/CNFs melt-spun nanocomposite filaments: (a) Tenacity and Initial modulus (b) elongation at break. 


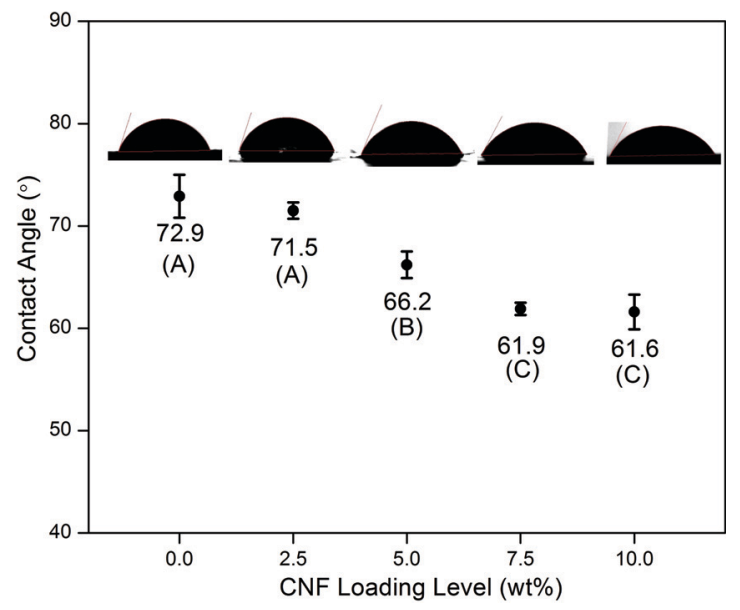

(a)

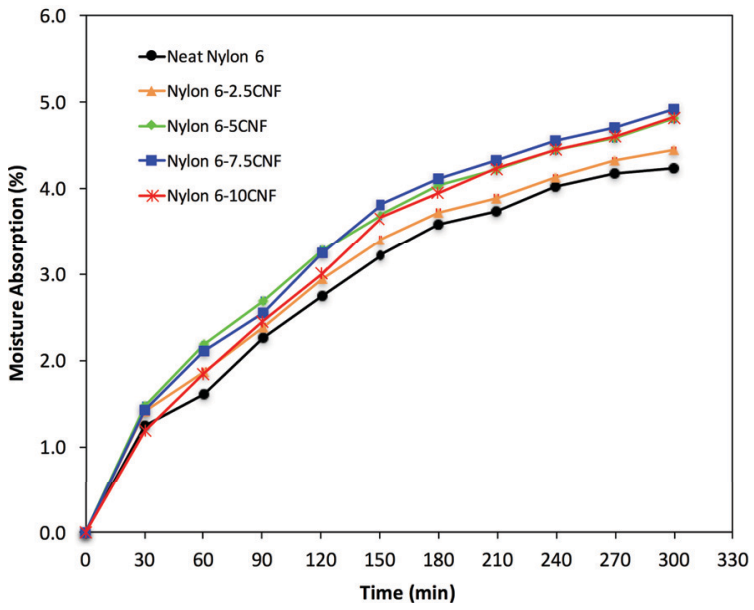

(b)

Figure 7. Water contact angles (a) and moisture absorption behavior (b) of Nylon 6/ CNFs nanocomposite films with respect to the CNF loading levels. 